University of Nebraska - Lincoln

DigitalCommons@University of Nebraska - Lincoln

Faculty Publications, Department of Physics and Astronomy

Research Papers in Physics and Astronomy

$9-11-2006$

\title{
Nonextensivity in magnetic nanoparticle ensembles
}

\author{
Christian Binek \\ University of Nebraska-Lincoln, cbinek@unl.edu \\ Srinivas Polisetty \\ University of Nebraska-Lincoln, polisetty.srinivas@gmail.com \\ Xi He \\ University of Nebraska-Lincoln, lodgehe@bigred.unl.edu \\ Tathagata Mukherjee \\ University of Nebraska-Lincoln, tatha.muk@gmail.com \\ Rajasekaran Rajesh \\ University of Nebraska-Lincoln \\ See next page for additional authors
}

Follow this and additional works at: https://digitalcommons.unl.edu/physicsfacpub

Part of the Physics Commons

Binek, Christian; Polisetty, Srinivas; He, Xi; Mukherjee, Tathagata; Rajesh, Rajasekaran; and Redepenning, Jody G., "Nonextensivity in magnetic nanoparticle ensembles" (2006). Faculty Publications, Department of Physics and Astronomy. 60.

https://digitalcommons.unl.edu/physicsfacpub/60

This Article is brought to you for free and open access by the Research Papers in Physics and Astronomy at DigitalCommons@University of Nebraska - Lincoln. It has been accepted for inclusion in Faculty Publications, Department of Physics and Astronomy by an authorized administrator of DigitalCommons@University of Nebraska Lincoln. 


\section{Authors}

Christian Binek, Srinivas Polisetty, Xi He, Tathagata Mukherjee, Rajasekaran Rajesh, and Jody G. Redepenning 


\title{
Nonextensivity in magnetic nanoparticle ensembles
}

\author{
Ch. Binek,* S. Polisetty, Xi He, and T. Mukherjee \\ Department of Physics and Astronomy and the Nebraska Center for Materials and Nanoscience, Ferguson Hall, University of Nebraska, \\ Lincoln, Nebraska 68588-0111, USA \\ R. Rajesh and J. Redepenning \\ Department of Chemistry and the Nebraska Center for Materials and Nanoscience, University of Nebraska, Lincoln, \\ Nebraska 68588-0304, USA
}

(Received 21 April 2006; revised manuscript received 29 June 2006; published 29 August 2006)

\begin{abstract}
A superconducting quantum interference device and Faraday rotation technique are used to study dipolar interacting nanoparticles embedded in a polystyrene matrix. Magnetization isotherms are measured for three cylindrically shaped samples of constant diameter but various heights. Detailed analysis of the isotherms supports Tsallis' conjecture of a magnetic equation of state that involves temperature and magnetic field variables scaled by the logarithm of the number of magnetic nanoparticles. This unusual scaling of thermodynamic variables, which are conventionally considered to be intensive, originates from the nonextensivity of the Gibbs free energy in three-dimensional dipolar interacting particle ensembles. Our experimental evidence for nonextensivity is based on the data collapse of various isotherms that require scaling of the field variable in accordance with Tsallis' equation of state.
\end{abstract}

DOI: $10.1103 /$ PhysRevB.74.054432

PACS number(s): 05.20.Gg, 65.80.+n, 82.60.Qr, 05.70.Ce

\section{INTRODUCTION}

Systems with long-range interactions cause important amendments to thermodynamics and statistical mechanics that are not yet fully explored. 1,2 Among them are the ensemble inequivalence ${ }^{3}$ and problems involved in the absence of a well-defined thermodynamic limit. ${ }^{4}$ Although these difficulties are widely recognized in the field of self-gravitating systems, for instance, ${ }^{5}$ they are also present but obtain less attention in three-dimensional (3D) magnetic dipolar interacting systems. In conventional bulk magnetic materials, quantum mechanical short-range exchange usually dominates the much weaker dipolar interaction. In accordance with this hierarchy of interactions, dipolar long-range contributions typically give rise to minor logarithmic corrections of the critical behavior 6 and contribute to the magnetic order only on a mesoscopic scale via domain formation.

Nowadays, advanced micromagnetic simulations successfully model the complex problem of ferromagnetic domain formation. ${ }^{7}$ The combination of micromagnetic methods on the mesoscopic scale with the canonical ensemble approach dealing with the statistical physics of short-range interactions is a very successful approximation, which masks, however, the fact that fundamental problems still challenge the concepts of thermodynamics and statistical mechanics. This becomes obvious when long-range interactions are the dominating forces in an ensemble of spins. Gross et al. suggest the exclusive use of the microcanonical ensemble when small systems or systems with long-range interactions or phase separations are studied. ${ }^{8}$ The necessity of this approach is justified when carefully reconsidering the foundation of the canonical ensemble, which is derived from the microcanonical ensemble. This derivation mandates extensivity of the energy, a requirement that is not met in the case of interacting magnetic dipoles in three dimensions. In fact, violation of ensemble equivalence between the microcanonical and the canonical ensemble has been shown explicitly in the antiferromagnetic mean-field $X Y$ model and in the infinite-range Blume-Emery-Griffiths model. ${ }^{9,10}$ In addition, breaking ergodicity in systems with long-range interactions can manifest themselves in unusual dynamic features, such as logarithmically diverging relaxation times. ${ }^{11}$ Extensive theoretical work exists in the field of long-range interacting systems, but there is a paucity of experimental work apart from recent attempts to measure the controversial nonextensive entropic parameter $q$ of Tsallis entropy. ${ }^{12,13}$

Magnetic systems serve as important benchmarks in the field of statistical physics. ${ }^{14}$ Superparamagnetic blocking and the impact of dipolar interaction on various types of collective magnetism, such as superspin-glass behavior and superferromagnetism, have been widely studied in magnetic single domain nanoparticles embedded in a nonmagnetic matrix, ${ }^{15-20}$ but none of these studies focused on the impact of long-range interaction on system size-dependent scaling. We believe that nanoparticle ensembles serve as interesting model systems for studying the influence of long-range interactions on fundamental thermodynamic quantities. The large magnetic moment of several thousand Bohr magnetons $\left(\mu_{B}\right)$ of magnetic single domain nanoparticles enhances the long-range particle-particle interaction in comparison with conventional atomic paramagnetic systems, even when atomic spacings are replaced by average particle distances of a few $10 \mathrm{~nm}$. A rough estimate suggests that two neighboring nanoparticles of $2 \times 10^{4} \mu_{B}$ separated by $80 \mathrm{~nm}$ have about a 20 times higher dipolar interaction energy than atomic moments of about $3 \mu_{B}$ separated by $0.6 \mathrm{~nm}$, which is similar to properties of the Co Tutton salt. Thus, we reasoned that ensembles of magnetic nanoparticle systems might serve as ideal candidates for the study of thermodynamics of longrange interacting systems. The Co Tutton salt is well known from adiabatic demagnetization applications. Despite being in a ${ }^{4} F$ spectroscopic state, the $\mathrm{Co}^{2+}$ ions in this salt have an effective spin $1 / 2$ in the crystal field, a parallel Landé-factor 


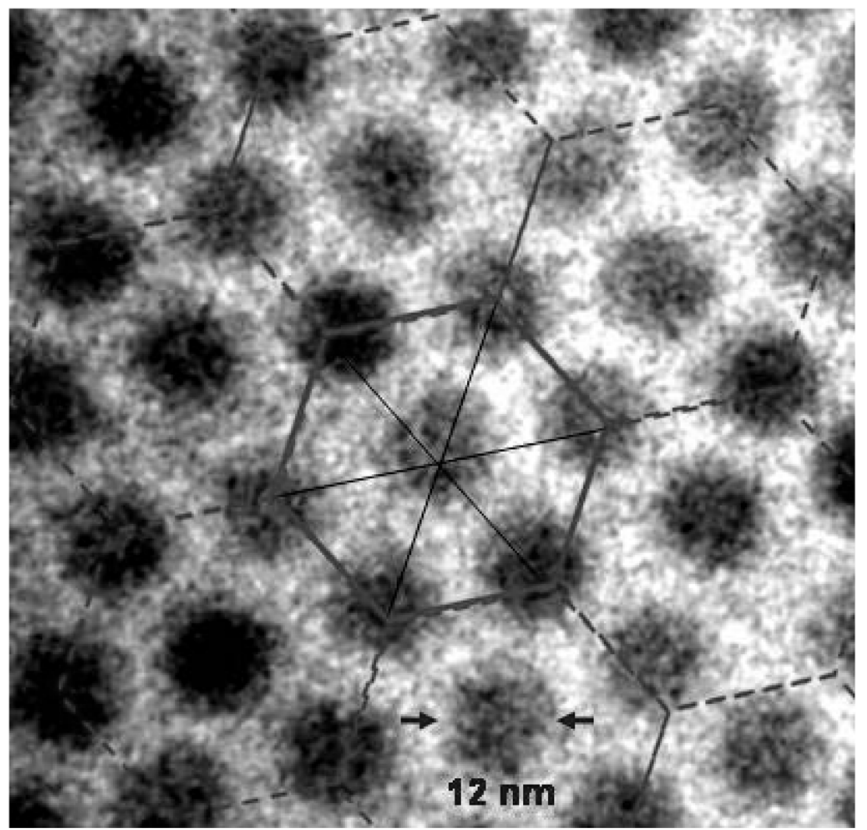

FIG. 1. Transmission electron microscopy image of a 2D selfassembled array of $\gamma-\mathrm{Fe}_{2} \mathrm{O}_{3}$ nanoparticles. The arrows indicate a particle diameter of $12 \mathrm{~nm}$. Eye-guiding lines stress the hexagonal dot arrangement controlled by the length of the organic molecules attached to the dot surfaces.

of $g=6.45$, and a nearest-neighbor spacing of $0.624 \mathrm{~nm} .{ }^{21}$

\section{SAMPLE PREPARATION}

Our samples are prepared using the technique of thermal decomposition of metal carbonyls in the presence of appropriate surfactants. The resulting nanoparticles are highly uniform and can be self-assembled into monolayers and multilayers. Such procedures were first developed by Thomas ${ }^{22}$ and were later popularized and refined by Puntes et al.,${ }^{23}$ and by Wang et al. ${ }^{24}$ Figure 1 shows a transmission electron microscopy (TEM) image of a typical two-dimensional (2D) self-assembled $\gamma-\mathrm{Fe}_{2} \mathrm{O}_{3}$ structure that we assembled from nanoparticles prepared using the carbonyl decomposition technique. In this example, self-assembling originates from geometrical constraints that are controlled by the length of the surfactant molecules attached to the surfaces of the $\gamma-\mathrm{Fe}_{2} \mathrm{O}_{3}$ nanoparticles. The length of the surfactant determines the particle-particle distance in a closed packed 2D structure, which is indicated in Fig. 1 by eye-guiding lines. The particle sizes follow a very narrow log-normal size distribution with an average diameter of $12 \mathrm{~nm}$. Very similar results are obtained for the 3D samples studied here where $\gamma$ - $\mathrm{Fe}_{2} \mathrm{O}_{3}$ nanoparticles produced by the same synthetic strategy are randomly dispersed in a polystyrene matrix such that the average spacing is much larger than the length of the surfactant. The particle sizes of the nanoclusters in our 3D samples have the mean diameter of $11.6 \mathrm{~nm}$. The standard deviation of the diameters is $0.3 \mathrm{~nm}$. These values are based on two different diameter measurements on each of 50 different nanoclusters. For these dilute 3D systems, the average particle separation is $\sim 80 \mathrm{~nm}$, which excludes any type of exchange mediated interaction between the particles. Hence, these systems are clearly governed by long-range dipolar interactions.

In two dimensions, the dipolar interaction energy per particle, $U(N, T=0) / N$, has an upper bound in the limit of a large particle number $N \rightarrow \infty$. The situation changes in 3D systems where $U(N, T=0) / N$ scales logarithmically according to $^{25-27}$

$$
\frac{U(N, T=0)}{N} \propto \int_{1}^{N^{1 / 3}} \frac{4 \pi r^{2}}{r^{3}} d r \propto \ln N .
$$

This simple result has various important implications. For example, a careful reconsideration of the thermodynamic limit $N \rightarrow \infty$ is required. The nonextensivity of the internal energy suggests that other thermodynamic quantitiesquantities normally considered to be extensive for systems dominated by short-range interactions-might no longer scale linearly with the sample size when 3D dipolar interactions are involved. From Eq. (1), it can be concluded that renormalization of $U / N$ allows one to recover convergence according to $U /(N \ln N) \rightarrow$ const for $N \rightarrow \infty$. Tsallis extended this reasoning to the Gibbs free energy, $G$. For a 3D system with dipolar interactions, Tsallis's scaling approach reads ${ }^{26,28}$

$$
\frac{G}{N \ln N}=\frac{U}{N \ln N}-\frac{T}{\ln N} \frac{S}{N}-\frac{\mu_{0} H}{\ln N} \frac{m}{N}+\frac{P}{\ln N} \frac{V}{N},
$$

where the entropy $S$, the magnetic moment $m$, and the volume $V$, scale with the system size $N$. The temperature $T$, the magnetic field $H$, and the pressure $P$, scale with $\ln N$, despite the fact that they are usually considered to be intensive variables in conventional thermodynamics treatments. For our magnetic nanoparticle system, this scaling implies an equation of state of the type ${ }^{28}$

$$
M=M\left(\frac{T}{\ln N}, \frac{H}{\ln N}\right),
$$

where $M=m / V$ is the magnetization of the sample.

The primary focus of the research effort described here is to provide experimental evidence regarding the validity of Eq. (3) by carefully examining 3D magnetic nanoparticle systems for which there are appreciable dipolar interactions. To check for the scaling behavior predicted by Eq. (3), we sought data collapse when plotting $M$ vs $(T / \ln N, H / \ln N)$ in accordance with the suggestion made by Tsallis. ${ }^{28}$

\section{EXPERIMENTAL RESULTS}

Figure 2 shows the typical temperature behavior of the magnetic moment $m$ after zero field cooling (ZFC) the sample and measuring $m$ vs $T$ on field heating [(FH), solid triangles] for temperatures $5 \leq T \leq 242 \mathrm{~K}$ in an applied field of $\mu_{0} H_{a}=25 \mathrm{mT}$. The subsequent field cooling curve [(FC), open triangles] separates from the FH branch at the blocking temperature $T_{B} \approx 127 \mathrm{~K}$, indicating the onset of nonequilibrium behavior. The fact that $m$ vs $T$ of the FC branch stays constant at $T \ll T_{B}$ establishes the absence of exchange inter- 


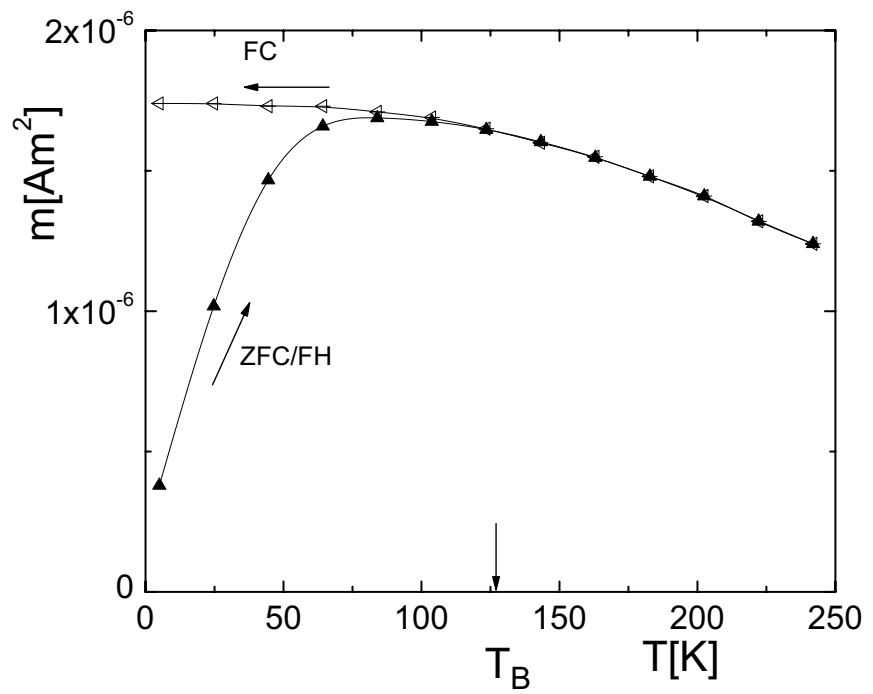

FIG. 2. $m$ vs $T$ of $\gamma-\mathrm{Fe}_{2} \mathrm{O}_{3}$ nanoparticles interacting magnetostatically in a random 3D array with an average particle spacing of $80 \mathrm{~nm}$. Solid triangles show the field heating curve (arrow labeled by $\mathrm{ZFC} / \mathrm{FH})$ in $\mu_{0} H_{a}=25 \mathrm{mT}$ after zero field cooling. Open triangles show the subsequent field cooling curve (arrow labeled by FC), which separates from the FH branch at the blocking temperature $T_{B} \approx 127 \mathrm{~K}$ (arrow). The lines connecting the data points are eye-guiding splines.

action that would otherwise modify the superparamagnetic blocking.

Superparamagnetic blocking is dominant when the time scale for thermally activated magnetization reversal of the nanoparticles exceeds the typical time scale of the measurement determined by our superconducting quantum interference device [(SQUID), Quantum Design MPMS XL-7]. In contrast to this simple freezing scenario, ferromagnetic (antiferromagnetic) exchange should cause an increasing (decreasing) $m$ vs $T$ dependence with decreasing temperature.

To overcome any experimental complications involving nonequilibrium behavior, we analyze only those magnetization isotherms $m$ vs $H$ that are measured at temperatures $T \gg T_{B}$. We compare three samples hereafter called samples $A, B$, and $C$. All samples have cylindrical shape with identical radii $R_{A, B, C}=2.64 \mathrm{~mm}$ and heights $h(A)=4.85, h(B)$ $=2.64$, and $h(C)=1.33 \mathrm{~mm}$. Hence, they built a descending sequence of volumes $V(A)=106.2, V(B)=57.8$, and $V(C)$ $=29.1 \mathrm{~mm}^{3}$. Magnetic fields were applied along the cylinder axis of the sample, respectively, and the applied fields, $H_{a}$, were corrected for demagnetizing fields according to $H$ $=H_{a}-N_{d} M$, where $H$ is the internal field, $M=m / V$ is the magnetization, and $N_{d}$ is the geometry dependent demagnetizing factor. Appropriate values for $N_{d}$ were calculated using the analytic result for oblate spheroids given by Osborn ${ }^{29}$

$$
N_{d}=\frac{\tilde{m}^{2}}{\tilde{m}^{2}-1}\left\{1-\frac{1}{\sqrt{\tilde{m}^{2}-1}} \arcsin \frac{\sqrt{\tilde{m}^{2}-1}}{\tilde{m}}\right\},
$$

which is a good approximation for our cylindrically shaped samples when using $\tilde{m}=2 R / h$. From Eq. (4), we obtain $N_{d}(A)=0.356, N_{d}(B)=0.527$, and $N_{d}(C)=0.702$. Note that SI

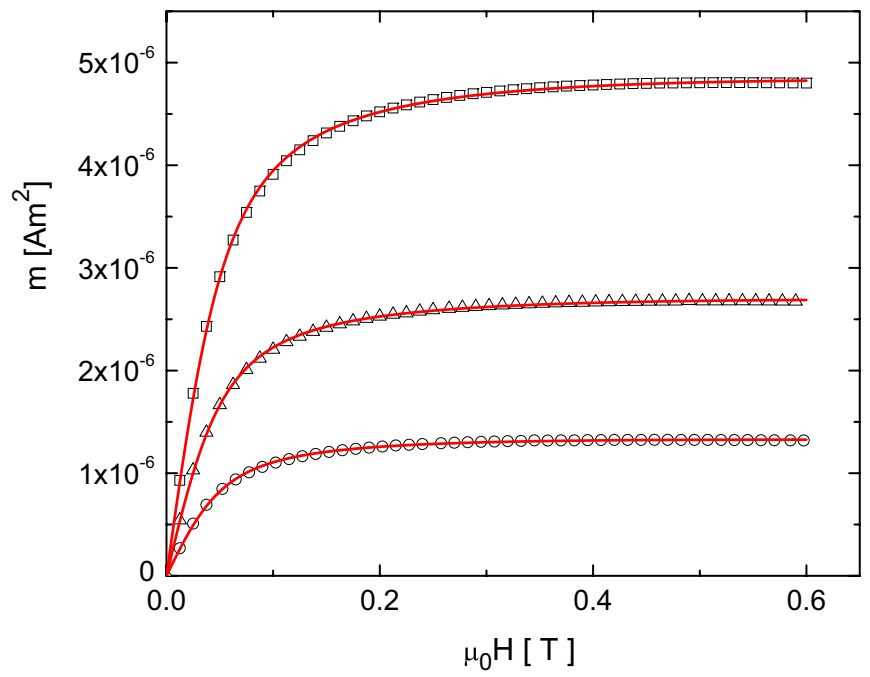

FIG. 3. (Color online) Isotherms $m$ vs. $H$ of sample $A$ (squares), $B$ (triangles), and $C$ (circles) measured at $T=300 \mathrm{~K}$, respectively. Note that only $1 / 5$ of the measured data points are shown. Lines are best fits of the Langevin function given by Eq. (5).

units are used where $0 \leq N_{d} \leq 1$ holds. The correction for demagnetizing fields is done to maintain a high level of accuracy, but it is important to note that for our system this correction is only a minor factor that changes the applied field by $<1 \%$.

Scaling according to Eq. (3) influences both the magnetic field and the temperature argument; however, when comparing two samples ( $B$ and $C$, for instance, which differ in size by a factor of about 2), the logarithmic dependence of the arguments of the scaling function (3) requires comparison of isotherms that differ in temperature by relatively small temperature changes of $\delta T / T=\ln 2 / \ln N$ where $N$ is on the order of $N \approx 10^{13}$. In terms of a zeroth order Taylor series $M\left(\frac{T_{C}}{\ln N_{C}}, \frac{H}{\ln N_{C}}\right)=M\left(\frac{T_{C}\left(1+\ln 2 / \ln N_{C}\right)}{\ln N_{B}}, \frac{\widetilde{H}}{\ln N_{B}}\right) \approx M\left(\frac{T_{C}}{\ln N_{B}}, \frac{\widetilde{H}}{\ln N_{B}}\right)$ of the first argument, we neglect scaling of the temperature but scale the magnetic field according to $\tilde{H}=H \ln N_{B} / \ln N_{C}$ when looking for a data collapse of the $m$ vs $H$ isotherms.

The influence of the dipolar interaction is expected to be quite small. Consequently, it is reasonable to describe the $m$ vs $H$ isotherms in zeroth-order approximation using the interaction free Langevin function

$$
m(H, T)=N m_{d o t}\left[\operatorname{Coth} \frac{\mu_{0} m_{d o t} H}{k_{B} T}-\frac{k_{B} T}{\mu_{0} m_{d o t} H}\right]
$$

to estimate the average magnetic moment $m_{d o t}$ of an individual nanoparticle. In addition, the Langevin fits with Eq. (5) provide the number of nanoparticles, $N_{A}, N_{B}$, and $N_{C}$, which are dispersed in our samples $A, B$, and $C$.

Figure 3 shows the results of the Langevin fits for all three samples at $T=300 \mathrm{~K}$. Within an uncertainty of $<3 \%$, the fits reveal the individual nanoparticle moment $m_{0}=2$ $\times 10^{-19} \mathrm{Am}^{2} \approx 2 \times 10^{4} \mu_{B}$. This is in satisfactory agreement with an estimation from geometrical considerations. We obtain the geometrical estimate of an individual nanoparticle moment of about $3 \times 10^{-19} \mathrm{Am}^{2}$ by assuming spherical nano- 
particles of $11.6 \mathrm{~nm}$ diam. We also assume bulk $\gamma-\mathrm{Fe}_{2} \mathrm{O}_{3}$ properties, including a density of $\rho=4.8 \mathrm{~g} / \mathrm{cm}^{3}$ and a saturation magnetization of $M_{s} \approx 74 \mathrm{Am}^{2} / \mathrm{kg} \cdot{ }^{30}$ The value of $m_{d o t}$ determined via the Langevin fit is $\sim 1 / 3$ smaller than the value obtained by geometrical estimation. This reduction is likely caused by a noncollinear spin structure at the particle surface, which, in turn, gives rise to partial spin compensation and, hence, a reduced moment. The qualitative agreement between the simple geometrical estimate and the more precise determination of $m_{d o t}$ via the fit is nevertheless a useful confirmation of the presence of single domain particles. It should be noted, however, that the geometrical estimation of $m_{d o t}$ does not affect the quantitative scaling analysis. The number of nanoparticles determined from the fits are $N_{A}=2.88 \times 10^{13}, N_{B}=1.5 \times 10^{13}$, and $N_{C}=7.8 \times 10^{12}$. These numbers are in good agreement with the volume ratios of the samples, e.g., $N_{B} / N_{C}=1.92 \approx V(B) / V(C)=1.98$. This agreement also demonstrates that the nanoparticles are distributed at a uniform concentration in all three samples.

Although the fits shown in Fig. 3 seem to be of satisfactory quality, closer inspection of the $m$ vs $H$ data shows that two isotherms corresponding to different sample sizes do not collapse when plotting $m / N$ vs $H$ or $M$ vs $H$. Such a data collapse would be suggested by Eq. (5) and, more generally, from conventionally expected extensivity of $m$ and intensitivity of $H$. The deviation from this conventional picture is visualized by plotting the ratio $m_{X} / m_{Y}$ vs $H$ for $X \neq Y$ and $X, Y=A, B, C$. The fact that $m_{X} / m_{Y} \neq$ const provides evidence for nonextensive behavior. Note that this statement holds also in the presence of a diamagnetic or more generally an extensive background signal. In addition, it is straightforward to show that deviations from a monodispersive distribution of particle sizes cannot account for $m_{X} / m_{Y} \neq$ const. Consider the thermal average of the total moment $m_{t o t}$ $=\sum_{i} N_{i} m_{i} L\left(\frac{\mu_{0} m_{i} H}{k_{B} T}\right)$ of noninteracting particles where $N_{i}$ specifies the number of particles with moment $m_{i}$ and where the sum includes all groups of particles with a given moment. The temperature and field dependence of the average moment of each individual species of nanoparticles is described by the Langevin function $L$ used in Eq. (5). When the sample volume is scaled by a factor $\alpha$, each $N_{i}$ is scaled according to $\tilde{N}_{i}=\alpha N_{i}$. The total average moment $\tilde{m}_{t o t}$ of a sample with the scaled volume is therefore given by $\widetilde{m}_{t o t}=\sum_{i} \tilde{N}_{i} m_{i} L\left(\frac{\mu_{0} m_{i} H}{k_{B} T}\right)$ $=\alpha m_{t o t}$. Hence, $\widetilde{m}_{t o t} / m_{t o t}=$ const holds for a distribution of nanoparticle sizes that is not necessarily monodisperse, while $m_{X} / m_{Y} \neq$ const originates from particle-particle interaction not included in the Langevin expression. Magnetostatic interactions are the only particle-particle interaction present in our samples; hence, magnetostatic interactions appear to be the origin of the nonextensive behavior that we observe.

Subsequently, we estimate the effect of magnetization inhomogenieties on the scaling analysis. Macroscopic measurements of the magnetization provide $M=M\left(H_{a}, T\right)$. Expressing the applied magnetic field in terms of the internal and the demagnetizing field, one obtains $M$ $=M\left[H_{a}\left(H, N_{d}, M\right), T\right]$. Here $N_{d}$ might be considered as an effective demagnetizing factor if inhomogeneous magnetization is taken into account. The relative change $\Delta M / M$ created by possible deviations $\Delta N_{d}$ from the geometrically de-
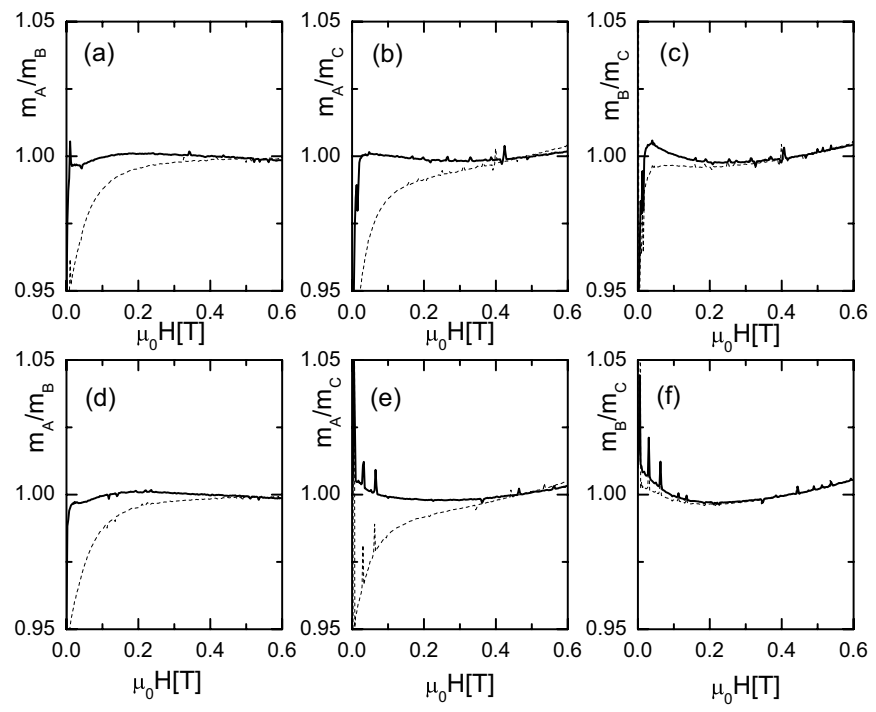

FIG. 4. Ratios $m_{X} / m_{Y}$ vs $H$ of the isotherms of samples $A, B$, and $C$ measured at $T=280 \mathrm{~K}$ (a)-(c) and $T=300 \mathrm{~K}$ (d)-(f), respectively. Dashed and solid lines represent the ratios without and with field-scaling.

termined demagnetizing factor $N_{d}$ can be estimated according to $\frac{\Delta M}{M} \approx \frac{\partial M}{\partial H_{a}} \frac{\partial H_{a}}{\partial N} \frac{\Delta N_{d}}{M}=\frac{\partial M}{\partial H_{a}} \Delta N_{d}$, where $\frac{\partial M}{\partial H_{a}} \leq \frac{\partial M}{\partial H}$. The susceptibility can be calculated either by directly differentiating the magnetization isotherms or, with even better accuracy, by differentiating Eq. (5). All parameters in Eq. (5) have been determined previously from the fits shown in Fig. 3. Differentiation of Eq. (5) and conversion of the total magnetic moment $m$ into the magnetization yields $\frac{\partial M}{\partial H}$ $=\frac{N m_{d o t}}{V}\left[\frac{k_{B} T}{\mu_{0} m_{d o t} H^{2}}-\frac{\mu_{0} m_{d o t}}{k_{B} T} \frac{1}{\sinh ^{2}\left(\mu_{0} m_{d o t} H / k_{B} T\right)}\right]$. This susceptibility expression rapidly reaches zero when $H$ approaches the saturation field. For our subsequent estimation of $\frac{\Delta M}{M}$, we consider the maximum susceptibility at $H=0$, which reads $\left.\frac{\partial M}{\partial H}\right|_{H=0}$ $=\frac{1}{3} \frac{N}{V} \frac{\mu_{0} m_{\text {dot }}^{2}}{k_{B} T}$. Even if we allow for unrealistically large fluctuations $\Delta N_{d} \leq 1$, we estimate $\frac{\Delta M}{M}<\frac{1}{3} \frac{N}{V} \frac{\mu_{0} m_{\text {dot }}^{2}}{k_{B} T}$. Substituting for instance the parameters for sample $B$ at $T=300 \mathrm{~K}$ yields $\frac{\Delta M}{M}<1 \times 10^{-3}$. This estimate of the maximum possible relative magnetization fluctuation is about two orders of magnitude smaller than the anomalies observed in Fig. 4. Hence, as outlined earlier in the text, the correction for demagnetization has a minor influence on the analysis of the data; in particular, fluctuations of the effective demagnetizing field due to magnetization inhomogeneities are an unlikely source of the anomalies apparent in Fig. 4. These anomalies are consistently interpreted in terms of nonextensive behavior. We will ultimately show (vide infra) that scaling of the field variable by a scaling factor $\gamma_{X, Y}=\ln N_{X} / \ln N_{Y}$ yields in fact $m_{X} / m_{Y} \approx$ const as predicted by Eq. (3).

\section{SCALING}

Instead of using the results $N_{A}, N_{B}$, and $N_{C}$ of the Langevin fits as input parameters for the scaling procedure, we determine the scaling factor by an independent least-squares fit analysis and compare the results of these independent ap- 
proaches. The least-squares analysis is an optimization for the collapse of two data sets $m_{X}$ vs $H$ and $m_{Y}$ vs $H$ obtained by minimizing

$$
F\left(\alpha, \gamma_{X, Y}\right)=\sum_{j}\left\{\alpha m_{X}[H(j)]-m_{Y}\left[\gamma_{X, Y} H(j)\right]\right\}^{2} .
$$

Here, $\alpha$ is a simple scaling factor that is used to adjust the amplitudes of the data sets according to the volume ratio of the samples; however, $\gamma_{X, Y}$ is the nontrivial scaling factor of the magnetic field. A value of $\gamma_{X, Y}$ close to the expected value of 1 indicates that corrections for the dipolar interaction are small. We take advantage of $\gamma_{X, Y} \approx 1$ through the simplifying first-order Taylor series

$$
m_{Y}\left(\gamma_{X, Y} H\right) \approx m_{Y}(H)+\frac{d m_{Y}(H)}{d H} H\left(\gamma_{X, Y}-1\right) .
$$

We obtain linear equations for $\alpha$ and $\gamma_{X, Y}$ by substituting expansion (7) into the functional (6) and applying the necessary conditions $\partial F / \partial \alpha=0$ and $\partial F / \partial \gamma_{X, Y}=0$ for the minimization of $F\left(\alpha, \gamma_{X, Y}\right)$. Their solution reads

$$
\gamma_{X, Y}=\frac{S_{Y^{\prime} H X}\left(S_{Y^{\prime} H X}-S_{Y X}\right)+S_{X X}\left(S_{Y^{\prime} H Y}-S_{Y^{\prime} H Y^{\prime} H}\right)}{S_{Y^{\prime} H X}^{2}-S_{X X} S_{Y^{\prime} H Y^{\prime} H}} .
$$

where $\quad S_{Y^{\prime} H X}=\Sigma_{j} \frac{d m_{Y}[H(j)]}{d H} H(j) m_{X}[H(j)], \quad S_{Y^{\prime} H Y}$ $=\sum_{j} \frac{d m_{Y}[H(j)]}{d H} H(j) m_{Y}[H(j)], \quad S_{Y^{\prime} H Y^{\prime} H}=\sum_{j}\left[\frac{d m_{Y}[H(j)]}{d H} H(j)\right]^{2}, \quad S_{Y X}$ $=\Sigma_{j} m_{Y}[H(j)] m_{X}[H(j)]$, and $S_{X X}=\Sigma_{j}\left(m_{X}[H(j)]\right)^{2}$.

Having calculated $\gamma_{X, Y}$, we obtain

$$
\alpha=\frac{S_{Y X}+\left(\gamma_{X, Y}-1\right) S_{Y^{\prime} H X}}{S_{X X}} .
$$

Figure 4 shows the ratios $m_{X} / m_{Y}$ vs $H$ of the isotherms of samples $A, B$, and $C$ measured at $T=280 \mathrm{~K}$ [Fig. 4(a)-4(c)] and $T=300 \mathrm{~K}$ [Fig. 4(d)-4(f)], respectively. Dashed and solid lines are the ratios without and with field scaling. All isotherm ratios are matched in amplitude by $\alpha=1.80,3.63$, and 2.01 [Fig. 4(a) $-4(\mathrm{c})$ ] and $\alpha=1.80,3.62$, and 2.02 [Fig. 4(d)-4(f)] obtained from Eq. (9), respectively. As expected, the $\alpha$ values are approximately given by the volume ratios or ratios of the numbers of nanoparticles of the samples as determined from simple geometry or the Langevin fits shown in Fig. 3.

In plots of $m_{X} / m_{Y}$ vs $H$ as shown in Fig. 4 (dashed lines), systematic deviations from $m_{X} / m_{Y}=$ const $\approx 1$ are a clear signature that the $m$ vs $H$ data of the various samples do not simply scale with the sample size or number of nanoparticles. Note that corrections due to the well-known shapedependent demagnetizing fields are already taken into account when considering $m$ vs $H$ isotherms instead of $m$ vs $H_{a}$ raw data. By scaling the field values of $m_{Y}$ vs $H$ by the scaling factors $\gamma_{A, B}=1.045, \gamma_{A, C}=1.062, \gamma_{B, C}=1.015$ for isotherms at $T=280 \mathrm{~K}$ and $\gamma_{A, B}=1.044, \gamma_{A, C}=1.050$, and $\gamma_{B, C}$ $=1.006$ for isotherms at $T=300 \mathrm{~K}$, we obtain $m_{X} / m_{Y}$ ratios that are significantly closer to $m_{X} / m_{Y}=$ const $\approx 1$ (Fig. 4 solid lines). These results strongly support the scaling law of Eq. (3) suggested by Tsallis ${ }^{28}$ and later observed in Monte Carlo simulations. ${ }^{26}$ Although field scaling gives rise to a signifi- cantly improved data collapse for $m_{A} / m_{B}$ vs $H$ and $m_{A} / m_{C}$ vs $H$ [Fig. 4(a)-4(e)], there is no significant improvement when considering $m_{B} / m_{C}$ vs $H$ [Fig. 4(c) and 4(f)]. The latter ratio involves the isotherms of the two thinnest samples, and the negligible impact of the field scaling might indicate a gradual crossover into 2D behavior. As pointed out in the Introduction, in the pure 2D case regular extensive behavior is expected even for dipolar interacting particles.

The field-scaling factors $\gamma_{X, Y}$ have been determined from Eq. (8) by optimizing the data collapse for each pair of isotherms $m_{X}$ vs $H$ and $m_{Y}$ vs $H$. The equation of state (3) independently implies $\gamma_{X, Y}=\ln N_{X} / \ln N_{Y}$. The latter relation allows for an independent calculation of the scaling factors from $N_{A}, N_{B}$, and $N_{C}$ determined via the Langevin fits that yield $\gamma_{A, B}=\gamma_{B, C}=1.022$ and $\gamma_{A, C}=1.044$ remarkably close to the scaling factors determined by independent optimization of the data collapse. In fact, $\gamma_{A, C}$ is the largest scaling factor in accordance with the fact that samples $A$ and $C$ have the largest difference in volume. Retroactive justification for not scaling the temperature is provided by the fact that deviations in $\gamma$ by $<1 \%$ are observed when optimizing the data collapse for isotherms measured at $T=280 \mathrm{~K}$ and $T=300 \mathrm{~K}$.

\section{FARADAY ROTATION}

Finally, to provide independent experimental support for the proposition that the scaling behavior [implied by Eq. (3) and substantiated in Fig. 4] originates from long-range interactions, we examined the Faraday rotation $\Theta$ vs $H_{a}$ of sample $A$ at room temperature using two different diameters of a laser beam probe. A polarized beam of an intensity stabilized laser diode $(\lambda=670 \mathrm{~nm})$ was transmitted through sample $A$ along its cylinder axis. The sample was mounted between the pole shoes of an electromagnet. An iris provided control over the diameter of the light beam that passed through the sample and the holes in the pole shoes of the electromagnet. The Faraday effect rotates the plane of the polarized light by an angle $\Theta$, an angle that is proportional to the magnetization of the sample. An analyzer aligned at $15^{\circ}$ with respect to the polarizer gives rise to a $\Theta$ dependence of the transmitted light intensity, which is detected by a photodiode. Using the Jones matrix formalism, we determine $\Theta$ vs $H_{a}$ from the measured field dependence of the light intensity.

In a magnetic system with short-range particle-particle interaction, the illuminated part of the sample can be considered as an effective sample volume that contributes to the Faraday signal. Particles outside this volume have no effect on the Faraday signal. Variation of the laser beam diameter can be considered equivalent to variation of sample diameter. If the magnetization is homogeneous, the Faraday rotation is determined by the thickness of the sample. If the same idea is transferred to an ensemble of nanoparticles that are coupled via long-range interactions, one would expect that the variation of the laser diameter between $d_{1}=3.37 \mathrm{~mm}$ and $d_{2}=1.97 \mathrm{~mm}$ is equivalent to the measurement of two cylindrical samples with diameters $d_{1,2}$. Furthermore, from the SQUID magnetometry, one might expect $\Theta\left(d_{1}\right) / \Theta\left(d_{2}\right)$ vs $H_{a} \neq$ const. A more careful consideration that takes into account the long-range interaction of the nanoparticles reveals 


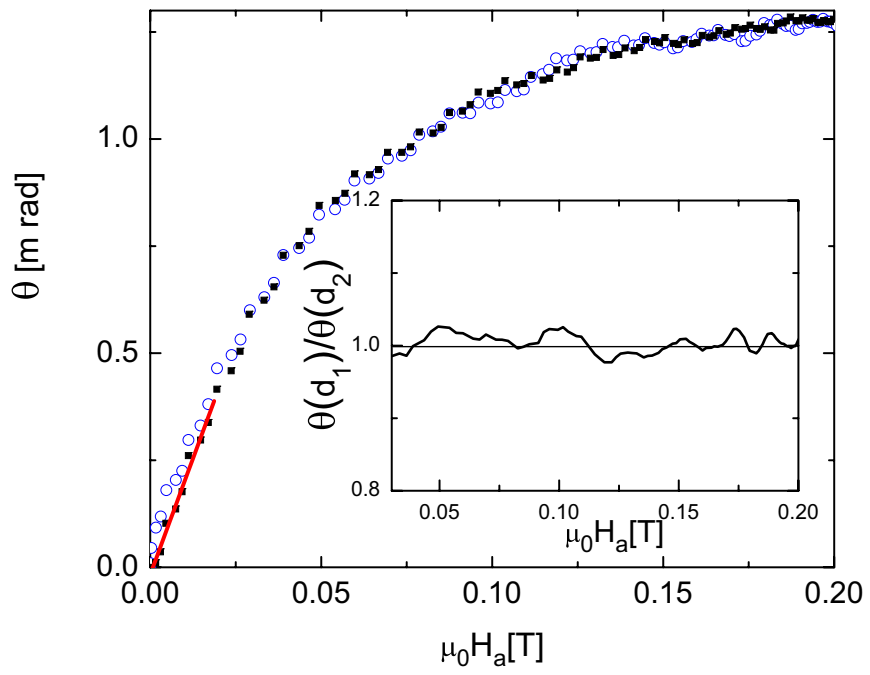

FIG. 5. (Color online) Faraday rotation $\Theta$ vs $H_{a}$ for the laser beam diameters $d_{1}=3.37 \mathrm{~mm}$ (squares) and $d_{2}=1.97 \mathrm{~mm}$ (circles). Solid line is a best linear fit to the $\Theta\left(d_{1}\right)$ vs $H_{a}$ data for $0 \leq \mu_{0} H_{a}$ $\leq 0.02 \mathrm{~T}$. The slope determines the Verdet constant of our $\gamma-\mathrm{Fe}_{2} \mathrm{O}_{3}$ nanoparticles dispersed in polystyrene. The inset shows the ratio of the data sets $\Theta\left(d_{1}\right)$ vs $H_{a}$ and $\Theta\left(d_{2}\right)$ vs $H_{a}$. The straight line indicates the expected ratio $\Theta\left(d_{1}\right) / \Theta\left(d_{2}\right)=1$ and visualizes the statistical fluctuations of the data.

that nanoparticles outside the illuminated region of the sample interact with nanoparticles within the laser spot, thus influencing the magnetization and the Faraday signal. Hence, the concept of an effective volume determined by the illuminated sample volume is not applicable in the case of longrange interaction. In short, $\Theta$ vs $H_{a}$ is expected to be independent of the diameter of the probing laser beam for a homogeneously magnetized sample.

Figure 5 shows the Faraday rotation $\Theta$ vs $H_{a}$ for the laser beam diameters $d_{1}=3.37 \mathrm{~mm}$ (squares) and $d_{2}=1.97 \mathrm{~mm}$ (circles). The slope of a best linear fit to the $\Theta\left(d_{1}\right)$ vs $H_{a}$ data for $0 \leq \mu_{0} H_{a} \leq 0.02 \mathrm{~T}$ provides the Verdet constant $V=4.54 \mathrm{rad} \mathrm{T}^{-1} \mathrm{~m}^{-1}$ where $\Theta=V \mu_{0} H_{a} h$. Similar results have been found recently when taking into account different saturation magnetizations for different nanoparticle concentrations. ${ }^{31}$ Within the noise level, the isotherms collapse as expected. The same holds for the corresponding ratios of the data sets shown in the inset. The ratio $\Theta\left(d_{1}\right) / \Theta\left(d_{2}\right)$ vs $H_{a}$ of the Faraday signals fluctuates statistically around $\Theta\left(d_{1}\right) / \Theta\left(d_{2}\right)=1$. The fact that the Faraday rotation signal is independent from the diameter of the probing laser beam is a retrospective indication for the absence of macroscopic heterogeneity across the sample. If present, such heterogeneity would diminish the reliability of the scal- ing analysis of our SQUID data. All samples are cut from a common master sample that is large in comparison to the individual samples $A, B$, and $C$.

Our study of the Faraday rotation emphasizes the fact that the dipolar interaction entangles all parts of the sample with each other. This entanglement also holds for those parts of the sample that are not directly probed by the laser beam, but a careful examination of isotherms for different sample volumes is required to assess the possible impact of long-range interactions on the magnetic equation of state. The independence of the Faraday-signal of the laser diameter provides no information about the nature of the interaction. The Faraday results are consistent with the presence of long-range interaction, but they offer no proof of nonextensive behavior.

\section{CONCLUSIONS}

In conclusion, we have provided experimental evidence for a magnetic equation of state, first proposed by Tsallis, which involves scaling the temperature and the magnetic field by the logarithm of the number of magnetic particles dispersed in the samples. We used SQUID magnetometry to measure the field dependence of the magnetic moment exhibited by various ensembles of magnetic $\gamma-\mathrm{Fe}_{2} \mathrm{O}_{3}$ nanoparticles embedded in a polystyrene matrix. We observe that the long-range nature of the dipolar interaction gives rise to the unusual scaling behavior of the isothermal magnetic moment measured as a function of the magnetic field. Our results substantiate Tsallis' approach concerning the phenomenology of nonextensive thermodynamics. When the magnetic field is scaled by the logarithm of the number of nanoparticles, the isotherms for different samples collapse to a common normalized result. The field-scaling factors that allow us to observe this collapse are determined by two independent methods that yield consistent results over the lengths scales to which we have experimental access. The applicability of similar unusual scaling procedures to various thermodynamic systems governed by an interaction range which is comparable to the characteristic length scale of the system should provide experimentalists and theorists with an interesting and exciting array of scientific challenges for many years to come.

\section{ACKNOWLEDGMENTS}

The authors gratefully acknowledge financial support through the Nebraska (MRSEC) Center for Quantum and Spin Phenomena in Nanomagnetic Structures. They are also pleased to thank R. D. Kirby for stimulating discussions and D. J. Sellmyer for enabling the SQUID measurements.

\footnotetext{
*Email address: cbinek2@unl.edu

${ }^{1}$ P. T. Landsberg, Thermodynamics and Statistical Mechanics (Oxford University Press, London, 1978); (Dover, New York, 1991), p. 102.
}

${ }^{2}$ D. Ruelle, Statstical Mechanics (Benjamin, New York, 1969).

${ }^{3}$ H. Touchette, R. S. Ellis, and B. Turkington, Physica A 340, 138 (2004).

${ }^{4}$ M. E. Fisher and D. Ruelle, J. Math. Phys. 7, 260 (1960). 
${ }^{5}$ T. Padmanabhan, Phys. Rep. 188, 285 (1990).

${ }^{6}$ A. Aharony, Phys. Rev. B 8, 3363 (1973).

${ }^{7}$ A. Hubert and R. Schäfer, Magnetic Domains (Springer, New York, 1998).

${ }^{8}$ D. H. E. Gross and J. F. Kenney, J. Chem. Phys. 122, 224111 (2005).

${ }^{9}$ T. Dauxouis, P. Holdsworth, and S. Ruffo, Eur. Phys. J. B 16, 659 (2000).

${ }^{10}$ J. Barré, D. Mukamel, and S. Ruffo, Phys. Rev. Lett. 87, 030601 (2001).

${ }^{11}$ D. Mukamel, S. Ruffo, and N. Schreiber, Phys. Rev. Lett. 95, 240604 (2005).

${ }^{12}$ M. S. Reis, V. S. Amaral, J. P. Araújo, and I. S. Oliveira, Phys. Rev. B 68, 014404 (2003).

${ }^{13}$ D. Jiulin, cond-mat/0602111, Europhys. Lett. (to be published).

${ }^{14} \mathrm{Ch}$. Binek, Ising-Type Antiferromagnets: Model Systems in Statistical Physics and in the Magnetism of Exchange Bias, STMP Vol. 196 (Springer-Verlag, Berlin, 2003).

${ }^{15}$ W. Kleemann, O. Petracic, Ch. Binek, G. N. Kakazei, Yu. G. Pogorelov, J. B. Sousa, S. Cardoso, and P. P. Freitas, Phys. Rev. B 63, 134423 (2001).

${ }^{16}$ J. L. Dormann, D. Fiorani, and E. Tronc, J. Magn. Magn. Mater. 202, 251 (1999).

${ }^{17}$ J. L. Dormann, D. Fiorani, R. Cherkaoui, E. Tronc, F. Lucari, D' Orazio, L. Spinu, M. Nogúes, H. Kachkachi, and J. P. Jolivet, J. Magn. Magn. Mater. 203, 23 (1999).
${ }^{18}$ C. Djurberg, P. Svedlindh, P. Nordblad, M. F. Hansen, F. Bodker, and S. Morup, Phys. Rev. Lett. 79, 5154 (1997).

${ }^{19}$ T. Jonsson, P. Svedlindh, and M. F. Hansen, Phys. Rev. Lett. 81, 3976 (1998).

${ }^{20}$ X. Chen, O. Sichelschmidt, W. Kleemann, O. Petracic, Ch. Binek, J. B. Sousa, S. Cardoso, and P. P. Freitas, Phys. Rev. Lett. 89, 137203 (2002).

${ }^{21}$ H. A. Farach, C. P. Poole, and J. M. Daniels, Phys. Rev. 188, 864 (1969).

${ }^{22}$ J. R. Thomas, J. Appl. Phys. 37, 2914 (1966).

${ }^{23}$ V. F. Puntes, K. Krishnan, and A. P. Alivisatos, Top. Catal. 19, 145-148 (2002).

${ }^{24}$ Z. L. Wang, Z. Dai, and S. Sun, Adv. Mater. (Weinheim, Ger.) 12, 1944 (2000).

${ }^{25}$ P. Jund, S. G. Kim, and C. Tsallis, Phys. Rev. B 52, 50 (1995).

${ }^{26}$ L. C. Sampaio, M. P. de Albuquerque, and F. S. de Menezes, Phys. Rev. B 55, 5611 (1997).

${ }^{27}$ F. Tamarit and C. Anteneodo, Phys. Rev. Lett. 84, 208 (2000).

${ }^{28}$ C. Tsallis, Fractals 3, 541 (1995).

${ }^{29}$ J. A. Osborn, Phys. Rev. 67, 351 (1945).

${ }^{30}$ V. Masheva, M. Grigorova, D. Nihtianova, J. E. Schmidt, and M. Mikhov, J. Phys. D 32, 1595 (1999).

${ }^{31}$ M. Zayat, F. del Monte, M. del Puerto Morales, G. Rosa, H. Guerrero, C. J. Serna, and D. Levy, Adv. Mater. (Weinheim, Ger.) 15, 1809 (2003). 